but individuals could not afford to purchase such equipment without a national subsidy in the first instance. The preferred alternative would be for the industrial countries to develop very simple designs of equipment which could be made in the country where required and thereby establish village industries. The first stage might well be along communal lines in villages, for baths, refrigeration and electricity generation, rather than attempting to accomplish these for individual dwellings. The importance of simplicity was emphasized, since in solar appliances the capital cost per unit of energy collected is more significant than the thermal efficiency of conversion. The sociological feature is the difficulty of introducing a new method of doing something already accomplished by other means, for example, to replace refuse-burning cookers by solar cookers, but where the application is completely new, as for electricity generation or refrigeration, there is not the resistance of tradition.

As regards industrial countries, solar energy can only be integrated with other energy sources, and water heating is the most feasible application. The prospects of success are greatest in the Mediterranean countries, decreasing but not necessarily being nonexistent in the more temperate countries. Much consideration was given to the assessment of potential resources in all the fields of energy considered. These data are particularly lacking as regards solar radiation and a much closer network of recording stations is required to assess the availability on a micro-climatic basis.

The final conclusions would appear to be:

Geothermal power is analogous to hydro-power, and obviously should be developed in the few localities where such sourees exist, provided that the power can be transmitted to centres of population. Wind- power is an established factor in agricultural areas. but development is needed before large wind-power generators can be constructed. Utilization of solar energy is the most difficult of all to establish. Protagonists must have faith in the possibilities, some of which are not yet proved. Similar situations have. however, arisen in other technologies now fully established and justified, for example, space travel. where the initial difficulties have eventually been overcome as unforeseen developments occurred. The urgent needs are international co-operation to establish satisfactory designs of equipment and the training in Europe and the United States of personnel from the user nations who can return to promote investigations in their own countries.

The general impression is that the development in six years is not as great as would have been expected. It is, however, encouraging to find that there are many people of high academic ability in various countries who have enough faith to devote their whole, or at least a considerable proportion, of their time to the attainment of a deeper knowledge of the underlying principles of all these sources of energy and in particular of solar energy devices, in order to raise the existing standard of living in under-developed countries. Co-ordinated planning during the next six years should promote a significant increase in applications.

Long-term predictions for the future are necessarily uncertain, but there is no doubt that energy demands will grow more rapidly than the world population. which is expected to double by the year 2000, and that all sources of energy should be actively investigated and integrated. The conference and discussions arising will undoubtedly promote these objectives. and lead to more successful international co-operation in future developments. H. HerwooD

\title{
MICROWAVE MEASUREMENT TECHNIQUES
}

\begin{abstract}
A CONFERENCE on the general subject of microwave measurements was held at the Institution of Electrical Engineers during September 6-8. A large audience of scientists and engineers from many countries assembled to consider some fifty papers covering all aspects of modern microwave measurement.

In his introductory lecture, Prof. H. E. M. Barlow, of University College, London, stated that microwave measurement techniques could, be grouped broadly under the following headings: measurements using field probes; measurements depending on resonance arrangements; attenuation and phase shift measurements; noise measurements and power measurements; and that the general limits of accuracy of microwave measurements at the present day were of the order of $\pm 0 \cdot 1$ per cent for impedance measurements, \pm 1 per cent for power measurements, and about $\pm 0.01 \mathrm{db}$. up to $3 \mathrm{db}$. for attentuation measurements.

Subsequent sessions of the conference considered most of these groups in detail.

Prof. Barlow also described two new devices utilizing the Hall effect for microwave power measurement. In the first, a thin indium arsenide crystal is rotated continuously in a wave-guide. The resulting Hall E.M.F. in the crystal is thus modulated at the
\end{abstract}

rotation frequency, unlike the parasitic thermoelectric and rectified E.M.F.s occurring at the crystal contacts. from which it can therefore be separated by a filter. The second device was demonstrated, and comprised an array of Hall crystals in cascade which could be used to measure high-power densities in free space. The multiplicity of crystals helps to cancel out the unbalanced rectifier effects occurring at the contacts.

A supporting paper by R. E. Larson of the Boulder Laboratories of the U.S. National Bureau of Standards outlined the calibration facilities offered by the Electronic Calibration Center of the Bureau. These are ultimately intended to cover the measurement of power, impedance, frequency, attenuation and noise over the full frequency-range $300-40,000 \mathrm{Mc}$. $\mathrm{s}$.

An interesting paper by Prof. C. Süsskind and P. O. Vogelhut, under the title "Cavity-perturbation Measurements of the Effect of Microwave Radiation on Proteins", described experiments concerned with the biological effects of microwave radiation on living animals. Irradiating power densities of the order of $0.1 \mathrm{~W} . / \mathrm{cm}^{2}$ produce significant changes of body temperature in mice, and accordingly the 'tolerance' level for human beings is accepted as $0.01 \mathrm{~W} . / \mathrm{cm}^{2}$. Apart from the heating effect, microwave irradiation causes other biological 
changes-in particular, it brings about a change in the hydration-rate of proteins. The measurement problem involved is one of determining the values of complex permittivities of the order of $60+30 j$, within times of the order of milliseconds. These are found by measurements of the $Q$ and resonant-frequency shift of a resonator through which the sample is passed at a constant rate.

J. E. Pearson and J. A. Jane of the Radio Research Station (Department of Scientific and Industrial Research) underlined the need for power density monitors to measure power densities of the order of 1-20 mW. $/ \mathrm{cm} .^{2}$ for health hazard purposes. Calibration of such meters requires a source of microwave energy of known power density and the authors described such a source utilizing parallel plate transmission line for frequencies between $0 \cdot 3$ and $1.5 \mathrm{Gc} / \mathrm{s}$.

Further papers presented on the first day dealt mainly with the measurement of impedance, admittance and reflexion coefficients, although papers dealing with noise measurement and problems of measurement associated with travelling wave tubes were also included. The former group of papers was largely concerned with the less-conventional types of measuring equipment: reflectometers; rotary standing wave indicators and 3-probe lines, together with an equipment for instantaneous impedance measurement described by $\mathrm{P}$. Hargrave and S. J. Robinson of Mullard Research Laboratories, which utilizes seven coaxial hybrid rings, capable of giving the polar components of an impedance directly to within \pm 10 per cent.

On the second day the subject of power measurement was reviewed in an introductory paper by Prof. A. L. Cullen of the University of Sheffield. The present position could be broadly summarized by stating that most instruments for the measurement of microwave power are accurate to within about \pm 2 per cent, although considerable difficulties still exist in providing reliable apparatus for the measurement of small powers, particularly at the higher frequencies. Among hitherto unutilized effects suggested by Prof. Cullen which might find application in future power-measuring devices were the use of electrostrictive and magnetostrictive materials to convert electromagnetic field energy into mechanical move. ment and the use of the saturation-level of maser-type devices, which occurs with increased pump-power, as a reference standard. A further similar scheme, using second-order saturation effects in ferrites, was put forward as being possible by Dr. P. J. B. Clarricoats of the Queen's University of Belfast. Prof. Cullen also suggested that it should be possible to make a wattmeter depending on the detection of the change of electromagnetic angular momentum occurring when circularly polarized waves are incident at a conical surface.

"A Survey of Microwave Power Measurement Techniques employed at the National Bureau of Standards (U.S.A.)" was presented by G. F. Engen, who dealt in detail with problems of bolometric measurement errors and mount efficiency.

J. A. Lane gave a "Comparison of Power Measuring Standards at Microwave Frequencies", indicating that measurements of the efficiency of numerous bolometer mounts conducted in various countries, whether determined by microcalorimeters, film bolometers, impedance measurement or torque vane wattmeters, were all in good agreement at power-levels of the order of 1-2 mW. The latter three methods exhibited errors generally of the order of \pm 1-2 per cent, while rather better accuracy was possible with the microcalorimeter.

An interesting new instrument for the measurement of power was described by Dr. E. Acs of Budapest. The power to be measured is absorbed in a film bolometer element terminating a waveguide and the consequent heating of the surrounding air causes air convection currents in an adjacent channel. The convected air stream impinges on a vane, the resulting deflexion of which is dependent on the power dissipated in the film.

Further papers by Prof. Barlow and D. Woods of the Ministry of Aviation described respectively a suggested method for the measurement of highfrequency power using a thin crystal of indium antimonide placed edge-on to the incident wave (it can be shown that in the absence of reflexions from the crystal edges the Hall voltage is directly proportional to the transmitted power), and a coaxial millivoltmeter/milliwattmeter which uses a silicon diode to measure voltage and power to within 0.5 per cent and 1.5 per cent, respectively, at all frequencies from d.c. to 1 Gc./s.

L. Lewin and G. H. B. Thompson of Standard Telecommunication Laboratories, Ltd., introducing the afternoon session, read a paper emphasizing the difficulty of "Measurements in Microstrip". The reasons for the present unsatisfactory state of affairs are first, the lack of a simple theory on the basis of which components can be designed and, secondly, no satisfactory insertion type detector yet exists.

Two papers presented by R. J. Turner of the Post Office Research Station (one in collaboration with D. L. Lisney) covered equipment used for the measurement of group delay and insertion loss of wave-guide networks in the 3.8-4.2 Mc./s. band for use in the testing of components for microwave links used for multi-channel telephony and television transmission. Other papers dealt with the measurement of waveguide attentuation, measurement of dispersion of disk-loaded wave-guide and of propagation coefficients in wave-guides with unknown but symmetrical input and output coupling. Among a group of papers devoted to attenuators, a paper on "Resistive Attenuators and their Calibration", by D. A. J. Walliker of Decca Radar, Ltd., dealt specifically with the use of metallized glass vanes and their mounts. Such attenuators with micrometer adjustment can yield accuracies of $0.04 \mathrm{db}$./micrometer division at 40-db. level in size 10 guide, with resetting accuracies up to an order of magnitude better than this. An attenuator of this type in size 16 guide has retained its calibration within $\pm 0.03 \mathrm{db}$. over a five-year period of laboratory use. Practical calibration systems for $S$ and $X$ band attenuators and their general specifications were also described.

The final morning was devoted specifically to millimetre wave techniques, the papers presented falling into two distinct groups, namely, those concerned with $H_{01}$ circular wave-guide transmission and those concerned with other topies. The former group included a paper by R. Hamer and R. J. Westcott of the Post Office Radio Research Laboratory on the messurement of attenuation in short lengths of circular guide. The $Q$ factor $\left(\sim 10^{6}\right)$ of a length of guide under test, when used as a resonator, was measured by comparing the resonator response curve when excited by a swept frequency oscillator with that of a standard reference resonator of lower $Q$. The response curves of the two resonators are matched by adjustment of the display sweep frequency and 
amplitude, the attenuation constant being deduced from the readings of the attenuators used to achieve this control. The ratio of measured to theoretical attenuation for plain copper guide and copper or aluminium helix guide was of the order of 1.4 at $35 \mathrm{Gc}$./s. for a 2 -in. diameter guide in all cases,

R. F. Skedd of the Standard Telephone Laboratories discussed the generation, and measurement of attenuation, of unwanted modes associated with long-haul wave-guides, while a paper by Dr. A. E. Karbowiak, G. Craven and V. H. Knight of the same establishment described an equipment for the automatic recording of wave-guide attenuation. This apparatus was capable of recording the variation of attentuation with frequency over the whole of the $40-80 \mathrm{Gc} . / \mathrm{s}$. band in a space of about $\frac{1}{2} \mathrm{hr}$.- an operation which, without the degree of automation used, would require some thousands of independent sets of measurements. An additional paper by Dr. Karbowiak and Mr. Skedd was concerned with an instrument for the accurate determination of relative surface losses in the millimetric band.

Among the other papers, Dr. J. Brown and Dr. A. P. Anderson of University College, London, dealt with the use of a single crystal as a harmonic generator and as a detector at a wave-length of $4 \mathrm{~mm}$. Such an arrangement with the addition of a simple ferrite modulator has been used to measure the impedance of wave-guide components at $4 \mathrm{~mm}$., as well as to determine the permittivity of liquid dielectrics.

W. L. Birch of the General Electric Co. Hirst Research Centre described a milliwattmeter capable of detecting changes of power $\sim 10 \mu \mathrm{W}$. for the 70-80 Gc./s. band in which the power to be measured is absorbed in a carbon losd, the temperature rise in which is detected by a thermopile with which it is in thermal contact. A further wattmeter for use at millimetric wave-lengths was demonstrated by Dr. H. A. French of A.S.W.E., Portsdown, and Prof. A. L. Cullen. The instrument was of the oscillating torque vane type, was capable of measuring power as low as $100 \mu \mathrm{W}$., and, like its predecessors, was also capable of absolute calibration.

The construction of Fabry-Pérot interferometers and their use for the measurement of permittivity of such substances as $\mathrm{TiO}_{2}$ was then considered in two papers by Dr. W. Culshaw and M. V. Anderson of General Telephone and Electronics Laboratories Inc., California, and by Dr. J. S. Seeley and J. C. Williams of Queen Mary College (University of London).

The final session was concerned with measurements on materials. Prof. Barlow described a method for the measurement of permittivity of high-loss dielectrics in which the specimen under test was located in an evanescent guide coupled to a resonant cavity, thus avoiding the disturbance of the field which normally results when the specimen is located within the cavity. For dielectries with small losses Prof. G. B. Walker and S. P. Luthra of the University of British Columbia used 'ghost modes' for the determination of complex permittivity. A disk specimen of the dielectric is located in a wave-guide and a resonant mode of the specimen-the 'ghost' modeis excited, the latter being a mode in which the fields outside the specimen are strongly evanescent and thus contain little stored energy. The limitations of the technique were discussed and a further paper by Prof. Walker and Messrs. J. M. Free and M. E. B. Moffat of the University of Oxford showed how these as well as normal cavity techniques could be used for the investigation of the properties of titanium dioxide and magnesium titanate ceramics at $S, X, J$ and $Q$ bands. Dr. Hamer and $\mathrm{Mr}$. Westcott described a method of measuring the complex permittivity of dielectric films while $R$. B. Nichols and A. E. Brown of Elliott Bros. spoke about an apparatus for grading dielectric cubes according to their exact permittivity. The cubes, used for the fabrication of Luneberg lenses, possess permittivities lying between 1 and 2 which are determined by noting the position at which resonance occurs, as a movable plunger sweeps the cube through a test cavity. Further papers by Dr. Clarricoats and by C. P. Aron of Morganite Research and Development, Ltd., described a simple wave-guide method for the determination of the permittivity of cylindrical dielectric rods and an apparatus for the measurement of the tensor susceptibility of ferrite materials.

G. D. Srms

\section{PROGRESS IN MICROCHEMICAL METHODS AND TECHNIQUES}

$\mathrm{T}$ HE 1961 International Symposium on Microchemical Techniques, organized by the Metropolitan Microchemical Society of the United States under the sponsorship of the Commission on Microchemical Techniques, Section of Analytical Chemistry, International Union of Pure and Applied Chemistry, was held during August 13-18, at Pennsylvania State University, University Park, Pennsylvania. The excellent accommodation and facilities made possible an informal atmosphere of fellowship and individual contacts. The purpose of the symposium was to provide a forum for the interchange of information and ideas among technologists from all parts of the world concerning new methods and techniques or unique applications of microchemical or microanalytical interest.

The symposium was inter-disciplinary both with respect to the interests of the almost five hundred technologists attending and the technical programme of ninety-five papers and lectures. The symposium was truly international in character with more than twenty countries represented. Travel of many speakers to the United States was supported by a generous research grant from the National Institutes of Health, U.S. Public Health Service.

The technical sessions and plenary lectures were supplemented by a three-day commercial exhibition of equipment, apparatus, specialities and literature, by six panel discussions, film sessions on the history of microchemistry and research advances, a practical demonstration session, a popular lecture, and by various social events.

The plenary lectures were of salient interest. $\mathrm{H}$ Lieb (Austria) delineated the history of microchemical techniques and A. A. Benedetti-Pichler (United States) the development of microchemistry in the United States. D. Van Slyke (United States) surveyed the evolution of micro-gasometric methods 